\title{
On the Relationship between Language Choice and Communicative Scope
}

\author{
ASTRID WESTERGAARD
}

This article discusses how multilingual young adults in the German minority in South Jutland use language on Facebook. I argue that language used on Facebook cannot be analyzed as a single phenomenon, but that the intended/addressed audience of 'friends' not only has a great influence on choosing between the available language codes, but also on whether and how the different language codes are used in specific situations. To describe the actual multilingual language use I distinguish between the language use in status updates and wall posts on Facebook. To explain the different choices made in language mode and code I argue that there is a connection between the type of Facebook post, the intended communicative scope, and the linguistic loyalty to macro or micro norms. Based on the data presented, I suggest a model for the analysis of multilingual language use in new media. 